\title{
Post-Vaccine Immunity against Hepatitis B in Burkina Faso children
}

\author{
SA Kissou ${ }^{1,2^{*}}$, K Sidibé $^{2}$, W Bazie ${ }^{3}$, Y Sourabié ${ }^{1,3}$, KR Cessouma $^{1,2}$, \\ AS Ouedraogo ${ }^{1,4}$, A Sawadogo $^{5}$ and B Nacro ${ }^{2}$ \\ ${ }^{1}$ Higher Institute of Health Sciences (INSSA); Nazi Boni University (UNB) of Bobo-Dioulasso, Burkina Faso \\ ${ }^{2}$ Department of Pediatrics, Souro Sanou University Hospital Center (CHUSS) of Bobo-Dioulasso, Burkina Faso \\ ${ }^{3}$ Immunology Laboratory, CHUSS, Burkina Faso \\ ${ }^{4}$ Laboratory of bacteriology-virology, CHUSS, Burkina Faso
}

${ }^{5}$ Department of gastroenterology and hepatology, CHUSS, Burkina Faso

Received: January 23, 2018; Accepted: February 26, 2018; Published: March 01, 2018

*Corresponding author: SA Kissou, Department of Pediatrics, Souro Sanou University Hospital Center (CHUSS) of Bobo-Dioulasso, Burkina Faso, Tel: + 226702476 34; E-mail: aimekissou@yahoo.fr

\begin{abstract}
Introduction: Hepatitis B is a major public health problem in the world. The routine immunization of infants against this disease has been effective in Burkina Faso for about ten years. The purpose of this study was to evaluate immunity against HBV in vaccinated children after a decade of vaccination.

Methods: a descriptive cross-sectional study was conducted; it included all children who had received at least one dose of HBV vaccine. A child was fully vaccinated if he had received all three doses of the vaccine. Assays for hepatitis B surface antigen (HBsAg) rates and anti-HBs antibody (anti-HBs) have been performed with the PLC ARCHITECT 4100. The latter threshold $10 \mathrm{IU} / \mathrm{L}$ for the title of anti-HBs antibody was considered to define a "good immunization". Logistic regression was used to determine the factors influencing the degree of immunization.
\end{abstract}

Results: 198 children were included with a sex ratio of 1.2 . Low birth weight was found in $10.40 \%$ of them. More than $30 \%$ of children received their first dose of vaccine between three and five months. Fully vaccinated children accounted for $89.4 \%$. The overall prevalence of HBsAg was $1 \%$ and that of the anti-HBs, $79.8 \%$. Eight out of ten children vaccinated were protected. The mean titer of the anti-HBs was $254,5 / \mathrm{L} \pm 329.1 \mathrm{UI}$. A multivariate analysis showed that only age (and thus decline compared to vaccination) was a factor significantly associated with optimal post-vaccination protection against hepatitis $B(p=0.011)$.

Conclusion: This study showed that the majority of children acquire protective anti-HBV immunity after primary vaccination according to the national immunization (Expanded Program of Immunization: EPI) regimen. However, the considerable decrease in the rate of anti-HBs after 3 years should perhaps be discussing the relevance of a booster vaccination within the epidemiological context and other risk factors.

Keywords: HBV vaccination; Immunization; EPI-Burkina Faso; children

\section{Introduction}

Low income countries are often highly endemic for hepatitis B. This is the case in Burkina Faso with a prevalence of hepatitis B surface antigen (HBsAg) of $14.5 \%$ [1]. This promotes perinatal and inter-infant transmission of hepatitis B virus (HBV). Infections are contracted at the age when the risk of chronic infection is highest. The routine infant immunization against the hepatitis B is recommended by the World Health Organization (WHO). In this context and for maximum effectiveness, the first dose should be given at birth [2].

Burkina Faso introduced HBV vaccine into the Expanded Program of Immunization (EPI) in 2006. It is combined with vaccines against diphtheria, tetanus, pertussis and hemophilus influenza b in a pentavalent vaccine. Ten (10) $\mu \mathrm{g}$ of HBsAg are contained in this combined vaccine. The primary vaccination starts at eight weeks of age and has three doses administered with one month interval. Booster vaccine is not recommended. Indeed, it is considered that $95 \%$ of infants are good responders to primary vaccination and that protection can last a good fifteen years. The decrease in the level of antibodies would not be a hindrance to this protection, because of the existence of immunity-memory [3].

The purpose of this work was to evaluate immunity against HBV in vaccinated children after a decade of vaccination in the country.

\section{Methods}

It was a descriptive transversal study. Data collection took place from February 15 to May 15, 2016 for a period of three months.

Was included in the study, children aged five months to 10 years old seen in the pediatric department of the Sanou Souro 
University Hospital Center (CHUSS) in Bobo-Dioulasso during the study period and receiving at least one dose of hepatitis $B$ vaccine. Accidental sampling was performed. Children with weakened fertility (HIV infection, malnutrition, polymal formative syndrome, immunosuppressive therapy) were not included.

A child was completely vaccinated if he had received all three doses of the vaccine. Were considered correctly vaccinated, those who were completely vaccinated, with a respect of the delays between the different doses.

A blood sample was taken on each child following the verification of his EPI vaccination card. Assays of HBsAg and anti-HBs antibody (anti-HBs) levels were performed on each sample. The analyses were carried out with the ARCHITECT 4100 automaton. The assay performed on the samples was quantitative.

The anti-HBs concentration of the sample was determined using an established ARCHITECT anti-HBs calibration curve (between 0 and $1000 \mathrm{IU} / \mathrm{L}$ ). When the concentration was greater than or equal to $10.0 \mathrm{IU} / \mathrm{L}$, the sample was considered reactive for anti-HBs. The threshold of $10 \mathrm{IU} / \mathrm{L}$ for the title of Ac anti-HBs was considered to define a good immunization.

The informed consent of the parents or guardians of the patients was required before any inclusion, through the signature of a consent form established for this purpose. Patients were also reassured about the confidential treatment of data, as well as the preservation of anonymity.

Data were coded and entered using Epi Data software version 3.1.1. They were analyzed using Stata software in version 12.0 . For comparison of proportions, Chi-square (x2), Pearson and Fischer tests were used. Kruskall Wallis test was used for means comparison. Logistic regression was used to determine the factors associated with good immunization. The significance level was $5 \%(p<0,05)$ with $95 \%$ confidence interval for all analyzes.

\section{Results}

A total of 198 children were included in the study, including 108 boys and 90 girls, a sex ratio of 1.2 . The average age was $19.3 \pm 16.2$ months. The under-3years were the most numerous (91.4\%).

The majority of the children had a normal birth weight. Low birth weight was found in $10.4 \%$ of them. Most children $(61.3 \%)$ received the first dose of the vaccine at two months of age, according to the EPI schedule in Burkina Faso. However, more than $38.7 \%$ of them received it beyond two months, some until the age of 9 months. Fully vaccinated children accounted for $89.4 \%$ of the total; $30.3 \%$ of the children were correctly vaccinated (Table I).

Regarding the determination of serological markers, 158 children were positive for anti-HBs (79.8\%) and 2 children for HBsAg among which, one positive for anti-HBs (Table II).

The overall prevalence of HBsAg was $1 \%$ and that of the antiHBs $79.8 \%$. The threshold for positivity of anti-HBs corresponded to the protective threshold of $10 \mathrm{IU} / \mathrm{L}$. Eight out of ten children vaccinated were protected. However, the antibody titer was variable, with an average titer of $254.5 \pm 329.1 \mathrm{IU} / \mathrm{L}$ and extremes of 10 and 1000 . There were $29.8 \%$ of children with a way of antiHBs between 10 and $100 \mathrm{U} / \mathrm{L}$ and 50\% above $100 \mathrm{IU} / \mathrm{L}$.

Table 1 : Distribution of children by number of doses of HBV vaccine received

\begin{tabular}{|c|c|c|}
\hline Number of doses & Proportion (\%) & Effective \\
\hline 01 & 3.5 & 07 \\
\hline 02 & 7.1 & 14 \\
\hline$\geq 03$ & 89.4 & 177 \\
\hline Total & $\mathbf{1 0 0}$ & $\mathbf{1 9 8}$ \\
\hline
\end{tabular}

Table 2 : Distribution of children by HBV status

\begin{tabular}{|c|c|c|c|}
\hline variables & HBsAg(+) & HBsAg(-) & Total \\
\hline anti-HBs (-) & $01(0.5 \%)$ & $39(19.7 \%)$ & $40(20.2 \%)$ \\
\hline anti-HBs (+) & $01(0.5 \%)$ & $157(79.3 \%)$ & $158(79.8 \%)$ \\
\hline Total & $02(1 \%)$ & $196(99 \%)$ & $198(100 \%)$ \\
\hline
\end{tabular}

In univariate analysis, antibody titer was associated with age at the time of assay, birth weight, and the number of vaccine doses received. Variables such as sex, age at first dose, and intervals between doses did not significantly influence antibody titer.

Tables III, IV and V show the variation of the titer of anti-HBs respectively depending on the age of children in dosage, birth weight and the number of received doses. The proportion of children immunized against hepatitis B decreased significantly with age $(p<0.01)$. Children less than 3 years were the best protected (Table III).

Table 3 : Distribution of children by age group and antibody titer

\begin{tabular}{|c|c|c|c|c|}
\hline \multirow{2}{*}{$\begin{array}{c}\text { Titeranti- } \\
\text { HBs(UI } \\
\text { / L) }\end{array}$} & \multicolumn{4}{|c|}{ Age group } \\
\hline & $\begin{array}{c}\text { Less than } 3 \\
\text { years }\end{array}$ & 3 to 5 years & $\begin{array}{c}\text { More than } 5 \\
\text { years }\end{array}$ & p-value \\
\hline Mean \pm SD & $254.6 \pm 322.7$ & $325.3 \pm 397.3$ & $1.8 \pm 3.1$ & 0,003 \\
\hline$<10, \mathrm{n}(\%)$ & $32(17.6)$ & 04 (30.8) & $04(100.00)$ & \\
\hline$\geq 10, \mathrm{n}(\%)$ & 149 (82.3) & $09(69.2)$ & $0(0.00)$ & \\
\hline $\begin{array}{c}\text { Total, n } \\
\text { (\%) }\end{array}$ & $181(100.00)$ & $13(100.00)$ & $04(100,00)$ & $<0.01$ \\
\hline
\end{tabular}

The lower the birth weight, the lower the titer. There was a statistically significant association between post-vaccination immunity and birth weight ( $\mathrm{p}=0.038$ ) (Table IV).

The antibody titer increased with the number of doses received. The association between the number of doses received and the immunological response to the HBV vaccine was statistically significant $(\mathrm{p}=0.014)$ (Table V).

In multivariate analysis, only age remained a factor significantly associated with optimal post-vaccination protection against hepatitis $B(p=0.011)$. Table VI gives the results of the multivariate analysis of the variables that were significant during the univariate analysis. 
Table 4 : Distribution of children by birth weight and antibody titer

\begin{tabular}{|c|c|c|c|c|}
\hline \multirow[t]{2}{*}{ Titeranti HBs(UI/L) } & \multicolumn{2}{|c|}{ Birth weight } & \multirow[b]{2}{*}{ Macrosomic } & \multirow[b]{2}{*}{ p-value } \\
\hline & Low & Normal & & \\
\hline Mean $\pm S D^{*}$ & $140.5 \pm 248.6$ & $251.8 \pm 321.8$ & $627.6 \pm 447.2$ & 0,038 \\
\hline
\end{tabular}

Table 5 : Variation of the antibody titer according to the number of doses received

\begin{tabular}{|c|c|c|c|c|c|}
\hline Titeranti-HBs(UI/L) & \multicolumn{4}{|c|}{ Number of doses received } & p-value \\
\hline Mean \pm SD* & 1 & 2 & 3 & 4 \\
\hline$<10 \mathrm{IU} / \mathrm{L}, \mathrm{n}(\%)$ & $29.2 \pm 49.7$ & $423.1 \pm 444.6$ & $252.7 \pm 318.7$ & $266.2 \pm 419.5$ & 0,035 \\
\hline$\geq 10, \mathrm{n}(\%)$ & $04(66.7)$ & $03(21.4)$ & $31(18.0)$ & $01(20.0)$ & $04(80.0)$ \\
\hline Total, $\mathrm{n}(\%)$ & $02(33.3)$ & $11(78.6)$ & $141(82)$ & $5(100)$ & 0.014 \\
\hline
\end{tabular}

* DS = Standard deviation

\begin{tabular}{|c|c|c|c|}
\hline \multicolumn{3}{|c|}{ Table VI: Multivariate analysisof factors associated with immune response to HBV vaccine } \\
\hline variables & Numbers & Adjusted OR (95\% CI) & p value \\
\hline & Age of the child & & 0,011 \\
\hline$\geq 3$ years & 181 & Reference population & 0.056 \\
\hline 2 years & 17 & $0.26(0,09-0,74)$ & \\
\hline & Birth weight & Reference population & \\
\hline$\geq 2500 \mathrm{~g}$ & 18 & $2.92(0.97$ to 8.79$)$ & 0.205 \\
\hline 1 dose & 155 & & \\
\hline 2 doses & Number of vaccines received & Reference population & \\
\hline 3 doses and more & 06 & $5.63(0.46$ to 68.17$)$ & $6.38(0.84$ to 48.53$)$ \\
\hline
\end{tabular}

\section{Discussion}

\section{Immunization profile of children}

Fully vaccinated children accounted for almost $90 \%$ of the total; this rate is globally satisfactory, since in 2016 , the global coverage was estimated at $84 \%$ [4]. However, the observance of the vaccination calendar remains insufficient. Indeed, the first dose that is recommended at eight weeks of life in the EPI of Burkina Faso was administered at this age to $61.3 \%$ of children. However, early onset of primary vaccination is of paramount importance in the prevention of perinatal transmission (vaccination at birth) and during infancy [2]. This is all the more important in a context of high prevalence of HBsAg carrier. In addition to the delay in the first vaccination, the delay between doses is not always respected, anything may hinder an optimal immune response.

\section{Serology}

The HBsAg was present in two children, both fully vaccinated. Whether transmission occurred during the perinatal period or during early childhood, it certainly could have been avoided by vaccination at birth. Indeed, this early vaccination at birth has a double benefit to avoid mother-to-child transmission of the virus on the one hand and to prevent transmission between children on the other. The prevention of infections in very young children allows a significant reduction in the chronic carriage of the virus in populations [5]. Vaccination at birth should be the rule in areas of high endemicity.

The anti-HBs were present in almost $80 \%$ of children in protective levels. Of fully immunized children, $82 \%$ were protected. The rest of the children could consist of poor immunization responders, and children who have progressively lost their antibodies but still have an amnestic immunity. The latter situation can be demonstrated by administering a booster dose that would lead to a rapid and important immune response [6]. The rapid implementation of a protective immune response through this memory immunity would also be effective during contact with the virus, preventing its development in the body. The progressive loss of antibodies over time after vaccination also largely explains the variation in the rate of anti-HBs in children. 
Almost 20\% had neither HBsAg nor anti-HBs. In the absence of anti-HBc antibody and viral DNA research, it is not possible to conclude on the situation of these children. In fact, occult hepatitis B cannot be ruled out in these cases. Additional analyzes have been recommended for these patients.

\section{Factors influencing the level of post-vaccination immunity}

Birth weight, number of doses and the age of children at the time of the evaluation were factors associated with the level of immunity assessed by the rate of anti-HBs.

Age at the time of evaluation (thus post-vaccination recession), was the first factor associated with the degree of immunity, in relation to the progressive decrease of antibodies after vaccination and over the years.

The low birth weight that is traditional during prematurity is accompanied by a fineness of the skin and a lack of muscle mass. This situation could hinder the administration or resorption of vaccines, the intramuscular and subcutaneous routes being the most used for vaccination. In the case of hepatitis $B$, the immunogenicity depends on a part of the birth weight (BW), on the other hand, from the postnatal age to the vaccination. The smaller the BW, the lower the seroconversion rate. This rate is less than $85 \%$ in less than 2000 grams $[7,8]$. In addition, seroconversion is optimal only around one month of life, hence the inefficacy of vaccination at birth, which is recommended to prevent vertical transmission of the virus in neonates of, mothers with HBsAg. The prevention of hepatitis B in these patients will include, in addition to vaccination, the administration of immunoglobulins [8]. In addition, the vaccination will include after the one given at birth, an additional dose to one month of life, from which a primary vaccination of at least four doses.

The number of doses received was the second factor associated with the degree of post-vaccination immunity. Indeed, a minimum of three doses was necessary for a protective immune response. This seems logical, since almost all immunization programs have three doses for primary immunization in children [6]. Nevertheless, a two-dose regimen is proposed by some for the vaccination of adolescents, with a rate close enough to good responders with three doses in infants; however, the decrease in the rate of anti-HBs would be faster [9].

\section{Conclusion}

After 10 years of routine infant immunization against hepatitis B virus in Burkina Faso, it appears that most children are protected against this disease. A larger scale study would be desirable to confirm this finding. Nevertheless, screening for carriage of HBsAg could be offered to pregnant women, so that neonates of those who are positive can benefit from a dose of vaccine at birth for optimal prevention.

\section{References}

1. Zampino R, Boemio A, Sagnelli C, Loredana Alessio, Luigi Elio Adinolfi, Evangelista Sagnelli, et al. Hepatitis B virus burden in developing countries. World J Gastroenterol. 2015;21(42):11941-11953. doi: 10.3748/wjg.v21.i42.11941

2. WHO. Introduction of the vaccine against Hepatitis B - Lines guidelines WHO / IVB / 01.31.

3. Bauer T, Jilg W. Hepatitis B surface antigen-specific T and B cell memory in individuals who had lost protective antibodies after hepatitis B vaccination. Vaccine. 2006;24(5):572-577.

4. WHO. Blanket vaccine. Available at http://www.who.int/mediacentre/ factsheets/fs378/fr/

5. Peto TJ, Mendy ME, Lowe Y, Webb EL, Whittle HC, Hall AJ. Efficacy and effectiveness of childhood immunization against chronic hepatitis B in the Gambia Hepatitis Intervention Study (1986-90) and in the nationwide immunization program. BMC Infect Dis. 2014;14:7. doi: 10.1186/1471-2334-14-7

6. Elke Leuridan, Pierre Van Damme . Hepatitis B and the Need for a Booster Dose. Clinical Infectious Diseases. 2011;53(1):68-75.

7. Arnaud Gagneur, Didier Pinquier and Caroline Quach. Hum Vaccin Immunother. 2015;11(11):2556-2563. doi: $10.1080 / 21645515.2015 .1074358$

8. Markus Waitz ,ReinhardHopfner , Helmut D. Hummler , U. Heininger . Hepatitis B Postexposure Prophylaxis in Preterm and Low-BirthWeight Infants. AJP Rep. 2015;5(1):e67-e72. doi: 10.1055/s-00351547329

9. Beran J, Kervyn D, Wertzova V, Hobzova L, Tichy P, Kuriyakose S, et al. Comparison of long-term (10 years) immunogenicity of twoand three-dose regimens of combined hepatitis A and B vaccine in adolescents. Vaccine. 2010;28(37): 5993-5997. doi: 10.1016/j. vaccine.2010.06.104 